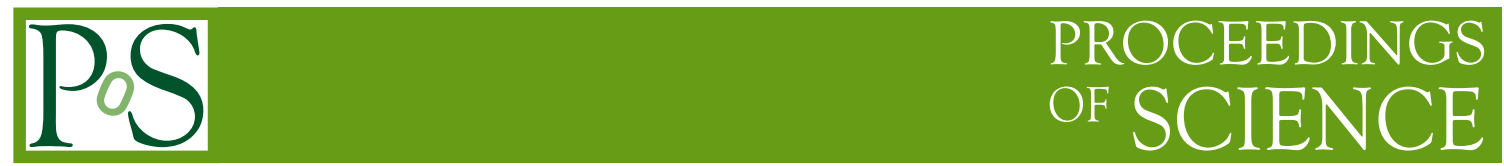

\title{
Recent CMS results on B hadron decays with charmonium
}

\author{
Aleksander Tulupov ${ }^{a, *}$ and Sergey Polikarpov ${ }^{a, b}$ \\ ${ }^{a}$ National Research Nuclear University “Moscow Engineering Physics Institute” (MEPhI), Moscow, Russia \\ ${ }^{b}$ The Lebedev Physical Institute of the Russian Academy of Sciences (LPI RAS), Moscow, Russia \\ E-mail: aleksander.tulupov@cern.ch
}

Using a data sample corresponding to an integrated luminosity of $103.7 \mathrm{fb}^{-1}$ of pp collisions, collected by the CMS experiment at $\sqrt{s}=13 \mathrm{TeV}$ in 2017-2018, the $\mathrm{B}^{0} \rightarrow \psi(2 \mathrm{~S}) \mathrm{K}_{\mathrm{S}}^{0} \pi^{+} \pi^{-}$and $\mathrm{B}_{\mathrm{s}}^{0} \rightarrow \psi(2 \mathrm{~S}) \mathrm{K}_{\mathrm{S}}^{0}$ decays are observed for the first time. Measurements of their branching fractions, relative to the $\mathrm{B}^{0} \rightarrow \psi(2 \mathrm{~S}) \mathrm{K}_{\mathrm{S}}^{0}$ decay, are presented.

*** The European Physical Society Conference on High Energy Physics (EPS-HEP2021), *** *** 26-30 July $2021 * * *$

*** Online conference, jointly organized by Universität Hamburg and the research center DESY ***

${ }^{*}$ Speaker 


\section{Introduction}

Starting from the observation of the exotic X(3872) [3] decaying into $J / \psi \pi^{+} \pi^{-}$, interest in $B$ hadron decays to final states containing a charmonium resonance has increased. The first charged tetraquark candidate, $Z(4430)^{+}$, was discovered in the $\psi(2 S) \pi^{+}$mass spectrum of the $B \rightarrow \psi(2 S) K \pi^{+}$decay $[1,2,4,6]$, and many other exotic states have been discovered afterwards [7].

This report presents a search [5] for the $B_{S}^{0} \rightarrow \psi(2 S) K_{\mathrm{S}}^{0}$ and $B^{0} \rightarrow \psi(2 S) K_{\mathrm{S}}^{0} \pi^{+} \pi^{-}$decays using a data sample of $103.7 \mathrm{fb}^{-1}$ of $p p$ collisions at $\sqrt{s}=13 \mathrm{TeV}$ collected by the CMS experiment in 2017 and 2018. Both decays can be used for CP violation measurements, while the $B^{0} \rightarrow \psi(2 S) K_{\mathrm{S}}^{0} \pi^{+} \pi^{-}$ decay also allows to search for exotic resonances in the intermediate decay products. The $\psi(2 S)$ and $K_{\mathrm{S}}^{0}$ mesons are reconstructed using the $\mu^{+} \mu^{-}$and $\pi^{+} \pi^{-}$channels.

\section{Event reconstruction and selection}

The $\psi(2 S) \rightarrow \mu^{+} \mu^{-}$candidates are reconstructed from muons passing the identification criteria. The $\psi(2 S) K_{\mathrm{S}}^{0}$ candidates are obtained by adding $K_{\mathrm{S}}^{0}$ candidates built from pions significantly displaced from the interaction region vertices corresponding to the $K_{\mathrm{S}}^{0} \rightarrow \pi^{+} \pi^{-}$decays. In case of $B^{0} \rightarrow \psi(2 S) K_{\mathrm{S}}^{0} \pi^{+} \pi^{-}$, additionally two oppositely charged tracks are included into the vertex fit of the $B$ meson candidate. In addition to the vertex fit quality, standard requirements are applied on the measured $B$ and $K_{\mathrm{S}}^{0}$ mesons decay lengths, in order to reduce the combinatorial background from prompt tracks.

\section{Observation of the decays}

The $\psi(2 S) K_{\mathrm{S}}^{0}$ and $\psi(2 S) K_{\mathrm{S}}^{0} \pi^{+} \pi^{-}$mass distributions are presented in Fig. 1 [5]. The signals of $B^{0}$ and $B_{s}^{0}$ mesons are described by a double Gaussian function with common mean, whose parameters are free in the unbinned maximum-likelihood fit. The combinatorial background is modelled with an exponential function. The $B_{s}^{0}$ signal on the left plot has the same shape as the $B^{0}$ signal, with the resolution parameters scaled by the ratio found in Monte-Carlo simulation (MC).

The contribution from $B_{S}^{0} \rightarrow \psi(2 S) K_{\mathrm{S}}^{0} K^{-} \pi^{+}$decay is accounted for the $m\left(\psi(2 S) K_{\mathrm{S}}^{0} \pi^{+} \pi^{-}\right)$fit by including a dedicated component with the shape derived from MC.

The ratio of signals in the $\psi(2 S) K_{\mathrm{S}}^{0}$ mass distribution, $N\left(B_{s}^{0}\right) / N\left(B^{0}\right)=(6.8 \pm 1.4) \cdot 10^{-3}$, is extracted directly from the fit, taking into account the correlation between the $B_{s}^{0}$ and $B^{0}$ yields, which are $113 \pm 23$ and $16660 \pm 140$, respectively [5]. The measured yield $N\left(B^{0} \rightarrow \psi(2 S) K_{\mathrm{s}}^{0} \pi^{+} \pi^{-}\right)$ is $3498 \pm 87$.

The significance of the $B_{s}^{0} \rightarrow \psi(2 S) K_{\mathrm{S}}^{0}$ decay is $5.2 \sigma$ and varies in the range 5.1-5.4 $\sigma$ within the variations of the fit model used in the estimation of the systematic uncertainties. The significance of the $B^{0} \rightarrow \psi(2 S) K_{\mathrm{S}}^{0} \pi^{+} \pi^{-}$signal exceeds 30 standard deviations [5].

The intermediate invariant mass distributions, corresponding to the signal four-body $B^{0} \rightarrow$ $\psi(2 S) K_{\mathrm{S}}^{0} \pi^{+} \pi^{-}$decay, are produced using the ${ }_{s} \mathcal{P}$ lot [8] technique to subtract the non- $B^{0}$ background. Obtained 2- and 3-body distributions are presented in Figures 2 and 3 [5]. No significant narrow peaks are seen in the $\psi \pi^{ \pm}\left(\pi^{\mp}\right)$ mass distributions. Signs of known $K^{*}(892)^{ \pm}, \rho(770)^{0}$, and $\mathrm{K}_{1}(1270)^{0}$ resonances are visible in the mass distributions of $K_{\mathrm{S}}^{0} \pi^{ \pm}, \pi^{+} \pi^{-}$, and $K_{\mathrm{S}}^{0} \pi^{+} \pi^{-}$, respectively. 

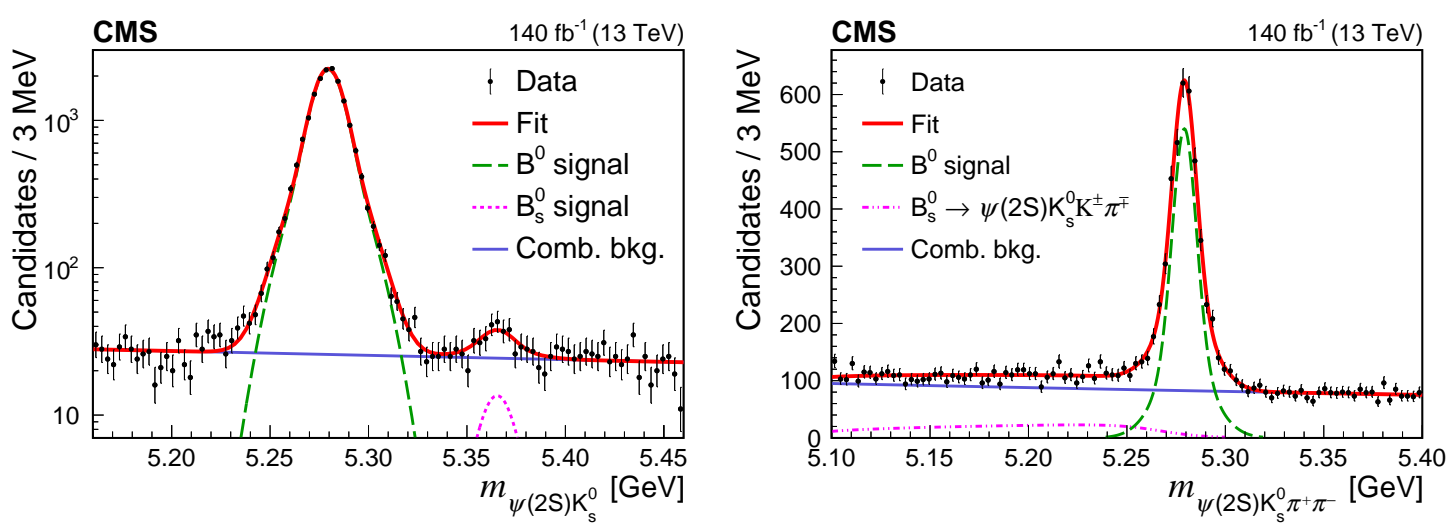

Figure 1: $\psi(2 S) K_{\mathrm{S}}^{0}$ (left) and $\psi(2 S) K_{\mathrm{S}}^{0} \pi^{+} \pi^{-}$(right) invariant mass distributions observed in data [5]. The green long-dashed line shows the $B^{0}$ signal, the blue solid line indicates the combinatorial background. The pink dashed line on the left figure shows the $B_{s}^{0} \rightarrow \psi(2 S) K_{\mathrm{S}}^{0}$ signal, and the pink dash-dotted line on the right figure shows the contribution from the $B_{s}^{0} \rightarrow \psi(2 S) K_{S}^{0} K^{ \pm} \pi^{\mp}$ decay, where the charged kaon was reconstructed as a pion.
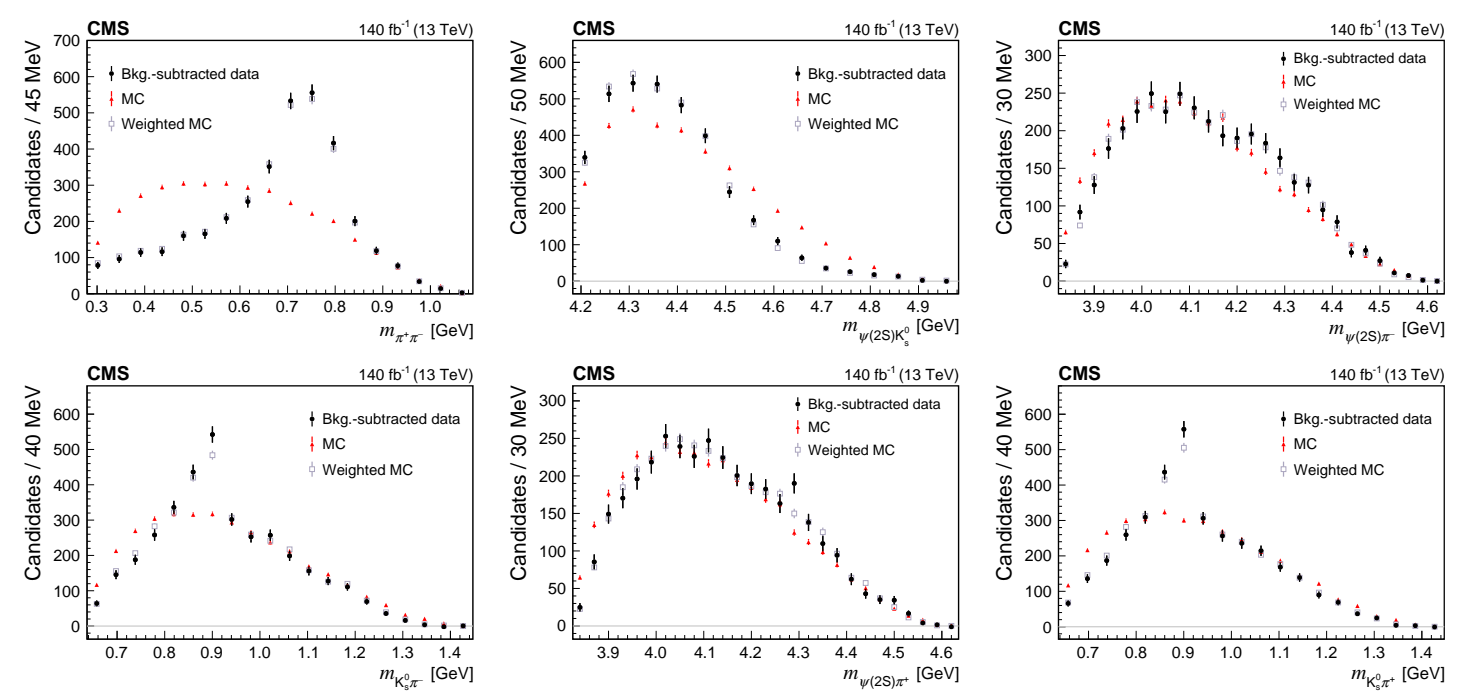

Figure 2: Distributions of 2-body intermediate invariant masses from the $B^{0} \rightarrow \psi(2 S) K_{\mathrm{S}}^{0} \pi^{+} \pi^{-}$decay [5]. Data distributions are background-subtracted, for MC only the statistical uncertainties are shown.

\section{Efficiencies and systematic uncertainties}

The relative branching fractions are measured using the expressions:

$$
\begin{aligned}
R_{S} \cdot \frac{f_{s}}{f_{d}} & \equiv \frac{\mathcal{B}\left(B_{s}^{0} \rightarrow \psi(2 S) K_{\mathrm{S}}^{0}\right)}{\mathcal{B}\left(B^{0} \rightarrow \psi(2 S) K_{\mathrm{S}}^{0}\right)} \cdot \frac{f_{s}}{f_{d}}=\frac{\epsilon\left(B^{0} \rightarrow \psi(2 S) K_{\mathrm{S}}^{0}\right)}{\epsilon\left(B_{s}^{0} \rightarrow \psi(2 S) K_{\mathrm{S}}^{0}\right)} \cdot \frac{N\left(B_{s}^{0} \rightarrow \psi(2 S) K_{\mathrm{S}}^{0}\right)}{N\left(B^{0} \rightarrow \psi(2 S) K_{\mathrm{S}}^{0}\right)}, \text { and } \\
R_{\pi^{+} \pi^{-}} & \equiv \frac{\mathcal{B}\left(B^{0} \rightarrow \psi(2 S) K_{\mathrm{S}}^{0} \pi^{+} \pi^{-}\right)}{\mathcal{B}\left(B^{0} \rightarrow \psi(2 S) K_{\mathrm{S}}^{0}\right)}=\frac{\epsilon\left(B^{0} \rightarrow \psi(2 S) K_{\mathrm{S}}^{0}\right)}{\epsilon\left(B^{0} \rightarrow \psi(2 S) K_{\mathrm{S}}^{0} \pi^{+} \pi^{-}\right)} \cdot \frac{N\left(B^{0} \rightarrow \psi(2 S) K_{\mathrm{S}}^{0} \pi^{+} \pi^{-}\right)}{N\left(B^{0} \rightarrow \psi(2 S) K_{\mathrm{S}}^{0}\right)},
\end{aligned}
$$



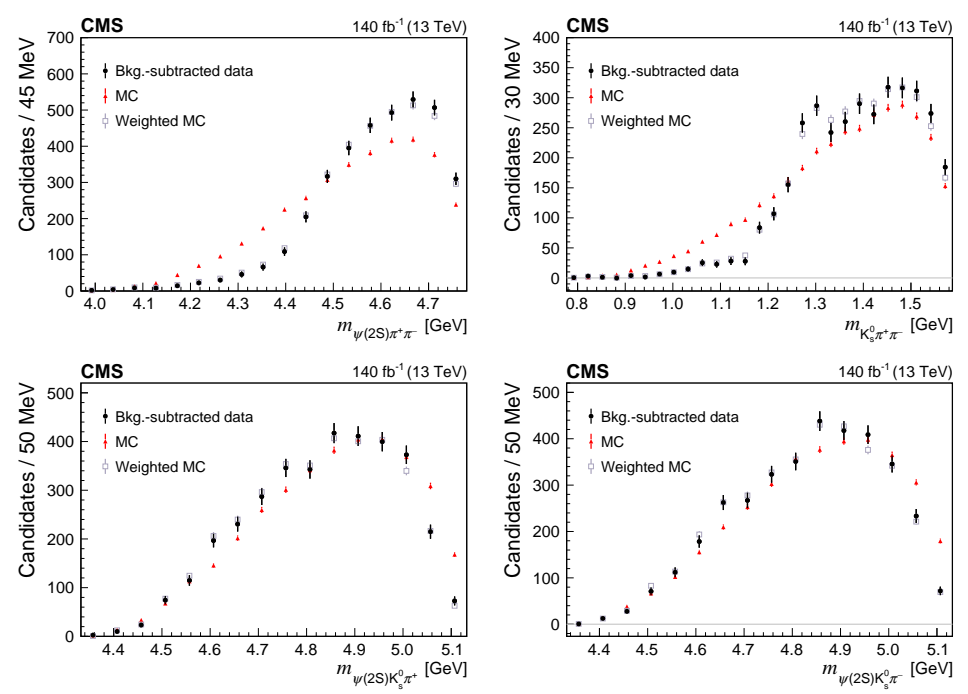

Figure 3: Distributions of 3-body intermediate invariant masses from the $B^{0} \rightarrow \psi(2 S) K_{\mathrm{S}}^{0} \pi^{+} \pi^{-}$decay [5]. Data distributions are background-subtracted, for MC only the statistical uncertainties are shown.

where $\epsilon$ is the total efficiency, $N$ is the number of reconstructed events in data, and $f_{d} / f_{s}$ is the ratio of production cross-sections of $B^{0}$ and $B_{s}^{0}$ mesons. The efficiency ratios are obtained using simulated event samples and are $1.02 \pm 0.01$ for the $R_{s} \cdot \frac{f_{s}}{f_{d}}$ measurement and $2.29 \pm 0.03$ for $R_{\pi^{+} \pi^{-}}$ measurement. The uncertainty related to the finite size of the MC samples (used to measure the efficiencies) is considered as a systematic uncertainty.

The systematic uncertainty related to the choice of the fit model is evaluated by testing different fit models, and the largest deviation in the measured ratio from the baseline value is taken as systematic uncertainty.

The uncertainty associated with the shape of the $B_{S}^{0} \rightarrow \psi(2 S) K_{\mathrm{S}}^{0} K^{-} \pi^{+}$contribution in the $\psi(2 S) K_{\mathrm{S}}^{0} \pi^{+} \pi^{-}$invariant mass is estimated by varying the shape parameters within their uncertainties.

The MC simulation does not take into account the intermediate resonance structure, leading to significant disagreement between data and MC in the intermediate mass distributions. To estimate the corresponding systematic uncertainty, the MC sample is reweighted to be consistent with the data, and the difference between the baseline efficiency and the efficiency obtained on the weighted $\mathrm{MC}$ is taken as a systematic uncertainty.

For the ratio $R_{\pi^{+} \pi^{-}}$, we consider an additional uncertainty due to tracking efficiency of 2 additional pions.

The total systematic uncertainties are $3.2 \%$ for $R_{s} \cdot \frac{f_{s}}{f_{d}}$ and $6.7 \%$ for $R_{\pi^{+} \pi^{-}}$. 


\section{Results}

The measured branching fraction of the $B_{S}^{0} \rightarrow \psi(2 S) K_{\mathrm{S}}^{0}$ decay, relative to the $B^{0} \rightarrow \psi(2 S) K_{\mathrm{S}}^{0}$ decay, multiplied by $f_{s} / f_{d}$ is [5]:

$$
R_{s} \cdot \frac{f_{s}}{f_{d}}=\frac{f_{s}}{f_{d}} \cdot \frac{\mathcal{B}\left(B_{s}^{0} \rightarrow \psi(2 S) K_{\mathrm{S}}^{0}\right)}{\mathcal{B}\left(B^{0} \rightarrow \psi(2 S) K_{\mathrm{S}}^{0}\right)}=(0.69 \pm 0.14(\text { stat }) \pm 0.02(\text { syst })) \%,
$$

where the first uncertainty is statistical, the second is systematic.

The branching fraction ratio of the $B^{0} \rightarrow \psi(2 S) K_{\mathrm{S}}^{0} \pi^{+} \pi^{-}$decay with respect to the $B^{0} \rightarrow$ $\psi(2 S) K_{\mathrm{S}}^{0}$ is [5]

$$
R_{\pi^{+} \pi^{-}}=\frac{\mathcal{B}\left(B^{0} \rightarrow \psi(2 S) K_{\mathrm{S}}^{0} \pi^{+} \pi^{-}\right)}{\mathcal{B}\left(B^{0} \rightarrow \psi(2 S) K_{\mathrm{S}}^{0}\right)}=(48.0 \pm 1.3(\text { stat }) \pm 3.2(\text { syst })) \% .
$$

\section{Summary and acknowledgments}

The first observation of the decays $B_{s}^{0} \rightarrow \psi(2 S) K_{\mathrm{S}}^{0}$ and $B^{0} \rightarrow \psi(2 S) K_{\mathrm{S}}^{0} \pi^{+} \pi^{-}$is reported, using pp collision data at $\sqrt{s}=13 \mathrm{TeV}$ taken by the CMS experiment [9] at the LHC. The two- and three-body invariant mass distributions of the $B^{0} \rightarrow \psi(2 S) K_{\mathrm{S}}^{0} \pi^{+} \pi^{-}$decay products do not show any exotics in addition to the known light meson resonances.

This work was supported by the Ministry of Science and Higher Education, project no. 07232020-0041 (Russia).

\section{References}

[1] AaIJ, R., ET AL. Observation of the resonant character of the $Z(4430)^{-}$state. Phys. Rev. Lett. 112 (2014), 222002.

[2] Chilikin, K., et Al. Experimental constraints on the spin and parity of the $Z(4430)^{+}$. Phys. Rev. D 88 (2013), 074026.

[3] Chol, S. K., ET AL. Observation of a narrow charmonium - like state in exclusive $B^{ \pm} \rightarrow$ $K^{ \pm} \pi^{+} \pi^{-} J / \psi$ decays. Phys. Rev. Lett. 91 (2003), 262001.

[4] CHOI, S. K., ET AL. Observation of a resonance-like structure in the $\pi^{ \pm} \psi^{\prime}$ mass distribution in exclusive $B \rightarrow K \pi^{ \pm} \psi^{\prime}$ decays. Phys. Rev. Lett. 100 (2008), 142001.

[5] CMS Collaboration. Observation of $\mathrm{B}^{0} \rightarrow \psi(2 \mathrm{~S}) \mathrm{K}_{\mathrm{S}}^{0} \pi^{+} \pi^{-}$and $\mathrm{B}_{\mathrm{s}}^{0} \rightarrow \psi(2 \mathrm{~S}) \mathrm{K}_{\mathrm{S}}^{0}$ decays. Tech. rep., CERN, Geneva, 2021.

[6] Mizuk, R., ET AL. Dalitz analysis of $B \rightarrow K \pi^{+} \psi^{\prime}$ decays and the $Z(4430)^{+}$. Phys. Rev. $D 80$ (2009), 031104.

[7] Particle Data Group, Zyla, P. A., et al. Review of particle physics. Prog. Theor. Exp. Phys. 2020 (2020), 083C01.

[8] Pivk, M., ANd Le Diberder, F. R. ${ }_{s}$ Plot: a statistical tool to unfold data distributions. Nucl. Instrum. Meth. A 555 (2005), 356.

[9] CMS Collaboration. The CMS experiment at the CERN LHC. JINST 3 (2008), S08004. 